\title{
Cerebral blood flow alterations specific to auditory verbal hallucinations in schizophrenia
}

\author{
Chuanjun Zhuo, Jiajia Zhu,* Wen Qin, Hongru Qu, Xiaolei Ma and Chunshui Yu
}

\section{Background}

Auditory verbal hallucinations (AVHs) have been associated with deficits in auditory and speech-related networks. However, the resting-state cerebral blood flow (CBF) alterations specific to AVHs in schizophrenia remain unknown.

\section{Aims}

To explore AVH-related CBF alterations in individuals with schizophrenia.

\section{Method}

In total, 35 individuals with schizophrenia with AVHs, 41 individuals with schizophrenia without AVHs and 50 controls underwent arterial spin labelling magnetic resonance imaging. The CBF differences were voxel-wise compared across the three groups.

\section{Results}

We found $\mathrm{AVH}$-specific $\mathrm{CBF}$ increase in the right superior temporal gyrus and caudate, and AVH-specific CBF decrease in the bilateral occipital and left parietal cortices. We also observed consistent CBF changes in both schizophrenia subgroups (i.e. those with and without AVHs) including decreased $\mathrm{CBF}$ in the bilateral occipital regions, the left lateral prefrontal and insular cortices, and the right anterior cingulate cortex and increased CBF in the bilateral lateral temporal regions and putamen, the left middle cingulate cortex and the right thalamus.

\section{Conclusions}

The AVH-specific CBF increases in the auditory and striatal areas and CBF reductions in the visual and parietal areas suggest that there exists a CBF redistribution associated with AVHS.

\section{Declaration of interest}

None.

\section{Copyright and usage}

(c) The Royal College of Psychiatrists 2017.
Auditory verbal hallucinations (AVHs) are one of the major positive symptoms of schizophrenia, affecting about $60-80 \%$ of individuals with schizophrenia. ${ }^{1}$ An AVH is an experience of spoken language without corresponding external stimulus. ${ }^{2,3}$ AVHs heavily affect the quality of life of individuals with schizophrenia, ${ }^{4}$ and up to $25 \%$ of AVHs are resistant to conventional treatments for schizophrenia. ${ }^{5}$ Therefore, a complete understanding of the underlying pathological process engaged in AVHs in schizophrenia may be critically important for developing specific and effective therapy, although different mechanistic models for AVHs have been proposed. ${ }^{6}$

A number of neuroimaging techniques have been utilised to investigate the neural substrates of AVHs in schizophrenia and have revealed structural and functional abnormalities in brain regions involving auditory processing, speech generation and perception. ${ }^{7,8}$ Structural imaging studies have demonstrated that AVHs are associated with grey matter volume reductions in brain regions involved in speech perception, such as the superior temporal gyrus (STG) and the primary auditory cortex. ${ }^{7-10}$ Diffusion tensor imaging studies have revealed white matter integrity disruption in the left arcuate fasciculus bundle connecting the frontal and temporal-parietal speech areas ${ }^{11}$ and in the corpus callosum fibres connecting the bilateral auditory regions ${ }^{12}$ in individuals with schizophrenia with AVHs. During the AVH state, functional magnetic resonance imaging (fMRI) has shown aberrant activation in the frontal-temporal speech areas ${ }^{8,13,14}$ and the primary auditory cortex ${ }^{15}$ in individuals with AVHs. Resting-state functional connectivity studies indicate that the core mechanism underlying AVHs involves a complex functional loop that is associated with speech areas. ${ }^{16}$

*These authors contributed equally to this work.
As the only currently available non-invasive perfusion imaging technique, arterial spin labelling (ASL) imaging has been used to investigate brain perfusion alterations in schizophrenia. ${ }^{17}$ Both increased and decreased resting-state cerebral blood flow (CBF) alterations have been found in individuals with schizophrenia. ${ }^{18-23}$ In a prior ASL study investigating perfusion changes associated with AVHs within an 'inner-speech' network, Wolf et al have observed increased CBF in the left STG and right temporoparietal cortex in individuals with AVHs compared with individuals without AVHs and controls. ${ }^{24}$ However, a complete picture of the whole-brain perfusion alterations specific to AVHs in schizophrenia beyond the speech-related network is yet to be unveiled. In this study, we used a $3 \mathrm{D}$ pseudo-continuous ASL technique to perform an exploratory analysis of AVH-related CBF alterations in schizophrenia by comparing voxel-wise CBF differences among individuals with schizophrenia with AVHs, individuals with schizophrenia without AVHs and healthy controls.

\section{Method}

\section{Participants}

A total of 126 right-handed individuals were enrolled in the present study, including 76 individuals with schizophrenia and 50 healthy controls. All participants were of Han Chinese ancestry. The diagnoses of schizophrenia were determined by the consensus of two experienced clinical psychiatrists using the Structured Clinical Interview for DSM-IV (SCID).${ }^{25}$ All healthy controls were screened using the non-patient edition of the SCID to confirm a lifetime absence of psychiatric illnesses. Exclusion criteria for all participants were a history of head trauma with consciousness disturbances lasting more than $5 \mathrm{~min}$, a history of drug or alcohol misuse, pregnancy and any physical illness such as cardiovascular disease or neurological disorders, as diagnosed by an interview 
and medical records review. In addition, all healthy controls were interviewed to exclude individuals with a known history of psychiatric illness in first-degree relatives. According to whether they had experienced AVHs, individuals with schizophrenia were subdivided into two groups: the AVH group $(n=35)$ that included individuals who experienced AVHs at least once daily, and the non-AVH group $(n=41)$ that included individuals who had never experienced AVHs or had not experienced AVHs within the 12 months prior to magnetic resonance imaging (MRI). Clinical symptoms of psychosis were quantified using the Positive and Negative Syndrome Scale (PANSS). ${ }^{26}$ The Auditory Hallucination Rating Scale (AHRS) ${ }^{27}$ was used to assess AVHs on seven characteristics: frequency, reality, loudness, number of voices, length, attention dedicated to the hallucinations and hallucination-induced arousal. Visual hallucinations were assessed using item six of the Scale for the Assessment of Positive Symptoms (SAPS). ${ }^{28}$ The item requires each individual with schizophrenia to answer if he/she has seen shapes or people that are not actually present. All individuals with schizophrenia had a rating of zero (no visual hallucinations) or one (questionable) on item six of the SAPS, suggesting that none of the participants with schizophrenia had a definite experience of visual hallucinations. The antipsychotic dosages are reported as chlorpromazine equivalents, which were calculated based on clinically equivalent dosing estimates. ${ }^{29}$ For each individual with schizophrenia, the chlorpromazine equivalent was estimated according to the antipsychotic drugs and dosages used in the last week before the MRI scan. Smoking habits were recorded in participants with schizophrenia, but the smoking status of the healthy controls was not known. Smokers were defined as individuals smoking currently and daily for more than 1 year any number of cigarettes and non-smokers were defined as individuals who did not currently smoke and had never smoked regularly or daily. ${ }^{30}$ The Medical Research Ethics Committee of Tianjin Medical University General Hospital approved this study. After a complete description of the study, written informed consent was obtained from each participant.

\section{MRI data acquisition}

The MRI scans were performed using a 3.0-Tesla MR system (Discovery MR750, General Electric, Milwaukee, Wisconsin, USA). Tight but comfortable foam padding was used to minimise head motion and earplugs were used to reduce scanner noise. The resting-state perfusion imaging was performed using a pseudocontinuous ASL sequence with a 3D fast spin-echo acquisition and background suppression (repetition time $(\mathrm{TR})=4886 \mathrm{~ms}$, echo time $(\mathrm{TE})=10.5 \mathrm{~ms}$, post-label delay $2025 \mathrm{~ms}$, spiral in readout of eight arms with 512 sample points; flip angle 111; field of view $($ FOV $)=240 \times 240 \mathrm{~mm}$; reconstruction matrix $128 \times 128$; slice thickness $4 \mathrm{~mm}$, no gap; 40 axial slices; number of excitations 3 ; and $1.9 \times 1.9 \mathrm{~mm}$ in-plane resolution). The total acquisition time for the resting-state ASL scan was 4 min $44 \mathrm{~s}$. During the scans, all participants were instructed to keep their eyes closed, relax and move as little as possible, think of nothing in particular and not fall asleep. All images were visually inspected to ensure that only images without visible artefacts were included in subsequent analyses.

\section{CBF calculation}

The ASL difference images were calculated using a singlecompartment model $^{31}$ after the subtraction of the label images from the control images. The CBF maps were subsequently derived from the ASL difference images and the proton-densityweighted reference images. ${ }^{32}$ Statistical Parametric Mapping $(\mathrm{SPM} 8)^{33}$ was used to co-register the CBF images of the 50 healthy controls to a positron emission tomography (PET)-perfusion template in the Montreal Neurological Institute (MNI) space using non-linear transformation. The standard CBF template of the MNI was defined as the mean co-registered CBF image of the 50 healthy controls. Then the CBF images of all participants were co-registered to the standard $\mathrm{CBF}$ template of the MNI and resampled to a voxel size of $2 \times 2 \times 2 \mathrm{~mm}$. Non-brain tissue was removed from each co-registered $\mathrm{CBF}$ map and spatially smoothed with a Gaussian kernel of $8 \times 8 \times 8 \mathrm{~mm}$ full-width at half maximum (FWHM). We normalised the CBF of each voxel by dividing the mean $\mathrm{CBF}$ of the whole brain. ${ }^{34}$

\section{Statistical analysis}

Group differences in CBF among the three groups were tested using a voxel-wise one-way analysis of covariance (ANCOVA) with age and gender as covariates followed by post hoc intergroup comparisons. The post hoc intergroup comparisons were conducted within a mask showing CBF differences from the ANCOVA analysis. Multiple comparisons were corrected using a false discovery rate (FDR) method with a significance threshold of $P<0.05$.

To investigate the relationship between CBF and the AHRS total score, a voxel-wise multiple regression analysis was conducted in the AVH group within regions showing significant CBF differences compared with the other two groups. The gender, age and antipsychotic dosages were considered nuisance covariates. Given the importance of the frequency of AVHs in neural correlations, ${ }^{35}$ we also performed the correlation between $\mathrm{CBF}$ and the frequency subscale of the AHRS. Multiple comparisons were corrected again using an FDR method.

\section{Results}

\section{Demographic and clinical characteristics}

Demographic and clinical data for the participants are presented in Table 1. The three groups were well-matched in terms of gender $\left(\chi^{2}=0.308, P=0.857\right)$ and age (one-way ANOVA, $F=0.100$, $P=0.905)$. There were no significant differences in antipsychotic dosages (two sample $t$-test, $t=1.163, P=0.248$ ), durations of illness (two sample $t$-test, $t=-0.916, P=0.363$ ), ratio of smoker/non-smoker $\left(\chi^{2}=1.484, P=0.223\right)$, PANSS negative score (two sample t-test, $t=-0.594, P=0.555$ ), PANSS general score (two sample $t$-test, $t=0.275, P=0.784$ ) and PANSS total score (two sample $t$-test, $t=0.893, P=0.375$ ) between individuals with and without AVHs.

\section{CBF differences across groups}

A voxel-wise ANCOVA revealed that the intergroup differences in $\mathrm{CBF}$ were mainly located in the bilateral lateral temporal, occipital and striatal regions, the left lateral prefrontal, parietal, middle cingulate and insular cortices, and the right anterior cingulate cortex (ACC) and thalamus (FDR corrected, $P<0.05$ ) (Fig. 1). Specifically, the individuals in the AVH group exhibited significantly decreased CBF in the bilateral occipital and left parietal regions and increased $\mathrm{CBF}$ in the right STG and caudate nucleus relative to the individuals in the non-AVH group (online Table DS1 and Fig. 1(b)). Compared with the control group, the $\mathrm{AVH}$ group showed significantly decreased CBF in the bilateral occipital regions, left lateral prefrontal, parietal and insular cortices and the right ACC and increased CBF in the bilateral lateral temporal regions and putamen, the left middle cingulate cortex (MCC) and the right thalamus (online Table DS2 and Fig. 1(c)). Similarly, the nonAVH group had significantly decreased CBF in the bilateral occipital regions, the left lateral prefrontal and insular cortices, and the right 


\begin{tabular}{|c|c|c|c|c|}
\hline Characteristics & $\begin{array}{l}\text { AVH group } \\
\quad(n=35)\end{array}$ & $\begin{array}{l}\text { Non-AVH group } \\
\qquad(n=41)\end{array}$ & $\begin{array}{l}\text { Control group } \\
\qquad(n=50)\end{array}$ & $P^{\mathrm{b}}$ \\
\hline Age, years: mean (s.d.) & $31.5(7.7)$ & $32.3(5.7)$ & $32.0(8.2)$ & 0.905 \\
\hline Gender, women/men: $n$ & $14 / 21$ & $17 / 24$ & $18 / 32$ & 0.857 \\
\hline Antipsychotic dosage, ${ }^{\mathrm{C}} \mathrm{mg} / \mathrm{d}$ : mean (s.d.) & $518.1(395.6)$ & $429.9(259.5)$ & $\mathrm{N} / \mathrm{A}$ & 0.248 \\
\hline Duration of illness, months: mean (s.d.) & $101.4(94.3)$ & $118.8(71.7)$ & $\mathrm{N} / \mathrm{A}$ & 0.363 \\
\hline Ratio of smoker/non-smoker, $n$ & $21 / 14$ & $30 / 11$ & $\mathrm{~N} / \mathrm{A}$ & 0.223 \\
\hline Positive and Negative Syndrome Scale, mean (s.d.) & & & $\mathrm{N} / \mathrm{A}$ & \\
\hline Total & $73.2(23.6)$ & $68.4(23.0)$ & & 0.375 \\
\hline Positive score & $20.1(7.7)$ & $14.8(7.6)$ & & 0.004 \\
\hline Negative score & $18.8(8.2)$ & $20.2(9.2)$ & & 0.555 \\
\hline General score & $34.3(11.5)$ & $33.6(10.5)$ & & 0.784 \\
\hline Auditory Hallucination Rating Scale total score, mean (s.d.) & $23.9(8.4)$ & & & \\
\hline \multicolumn{5}{|c|}{$\begin{array}{l}\text { N/A, not applicable. } \\
\text { a. All participants are of Han Chinese ancestry. } \\
\text { b. One-way ANOVA was used to test the difference in age across the three groups. Chi-square test was used to test the difference in gender across the three groups. Two-sample } \\
t \text {-test was used to compare the differences in antipsychotic dosage, duration of illness and PANSS scores between the two schizophrenia groups. Chi-square test was used to test } \\
\text { the difference in the ratio of smokers/non-smokers between the two schizophrenia groups. } \\
\text { c. Antipsychotic dosages are reported as chlorpromazine equivalents calculated based on clinically equivalent dosing estimates. }\end{array}$} \\
\hline
\end{tabular}

ACC and increased CBF in the bilateral lateral temporal regions and putamen, the left MCC and the right thalamus compared with the control group, but the spatial extent was smaller than that in the individuals in the AVH group (online Table DS3 and Fig. 1(d)).

Notably, both the schizophrenia groups showed an overlapping reduction of $\mathrm{CBF}$ in the bilateral occipital regions, the left lateral prefrontal and insular cortices, and the right ACC and an overlapping increase of $\mathrm{CBF}$ in the bilateral lateral temporal regions and putamen, the left MCC and the right thalamus, which were defined as common $\mathrm{CBF}$ alterations shared by individuals with and without AVH (Fig. 2(a)). Compared with both the non-AVH and control groups, the AVH group exhibited decreased CBF in the bilateral occipital and left parietal regions and increased $\mathrm{CBF}$ in the right STG and caudate, which were defined as exclusive CBF alterations in individuals with AVH (Fig. 2(b)).

\section{Associations between CBF and AVH severity in the AVH group}

In brain regions demonstrating AVH-exclusive CBF alterations, we did not find any significant correlation between $\mathrm{CBF}$ and $\mathrm{AVH}$ severity (both ARHS total score and ARHS frequency subscale) in the AVH group.

\section{Discussion}

\section{Main findings}

In this study, we adopted a 3D-ASL approach to study the wholebrain CBF patterns in individuals with schizophrenia with AVHs, individuals with schizophrenia without AVHs and healthy controls. Although the two schizophrenia groups showed widespread common CBF alterations in the frontal, temporal, occipital, insular and subcortical regions, the AVH group exhibited exclusive alterations, including increased $\mathrm{CBF}$ in the STG and caudate nucleus, and decreased CBF in the occipital and parietal cortices.

\section{Common CBF alterations in individuals with schizophrenia with and without AVHs}

Our findings of decreased CBF in the ACC, lateral prefrontal cortex, insular and occipital cortices and increased CBF in the lateral temporal cortex, putamen and thalamus in both schizophrenia groups provide further evidence that $\mathrm{CBF}$ impairments in these regions may be at the root of pathogenesis of schizophrenia, which was consistent with our prior study. ${ }^{23}$ The hypoperfusion in the lateral prefrontal cortex, the salience network (including the dorsal ACC and the anterior insular) and the visual region may contribute to deficits of cognitive processing, ${ }^{36}$ switching between central-executive and defaultmode networks ${ }^{37}$ and visual processing ${ }^{38}$ in schizophrenia. The lateral temporal cortex, thalamus and putamen are involved in processes of multimodal sensory integration, modulation of multiple functional circuits and dopamine function; hyperperfusion in those regions may lead to disturbances in their functions in schizophrenia. ${ }^{39-41}$ Thus, perfusion abnormalities in these brain circuits may contribute to the occurrence of schizophrenia irrespective of the presence of AVHs.

\section{CBF alterations specific to AVHs in schizophrenia}

We found that individuals with AVHs showed CBF increases in the right STG and caudate nucleus when compared with individuals without $\mathrm{AVH}$ and healthy controls, these findings are inconsistent with a previous CBF study within the 'inner-speech' network showing that the increased $\mathrm{CBF}$ in the left STG and right temporoparietal cortex were specific to AVHs in schizophrenia. ${ }^{24}$ The discrepancy may be related to differences in multiple aspects between the two studies: sample size (35 individuals with AVHs and 41 individuals without AVHs in our study v. 10 individuals in each group in Wolf et al's study), schizophrenic subtypes (mixed subtypes $v$. paranoid subtype), gender (matched $v$. unmatched between groups), ASL techniques (3D-pseudocontinuous ASL $v$. continuous ASL) and correction for multiple comparison (corrected $v$. uncorrected). Sommer and colleagues have noted that the right homologues of the language areas (including the right STG), rather than the language areas in the left hemisphere, were mainly activated while individuals with schizophrenia experienced AVHs in a scanner. ${ }^{42,43}$ In an early fMRI report, Woodruff et al have found that AVHs predominantly activate the right language areas including the right temporal cortex, whereas auditory perception of speech activates the left ones. ${ }^{44}$ Although several structural imaging studies have reported that individuals with schizophrenia with AVHs exhibit decreased volume $^{8,9}$ and cortical thickness ${ }^{45}$ in the bilateral STG, a metaanalysis has reported that there is a significant negative correlation between the severity of auditory hallucinations and grey matter 

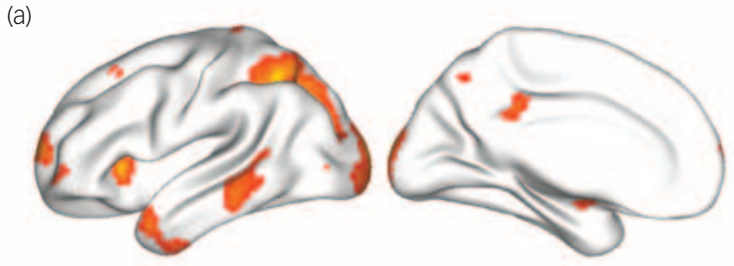

5.75

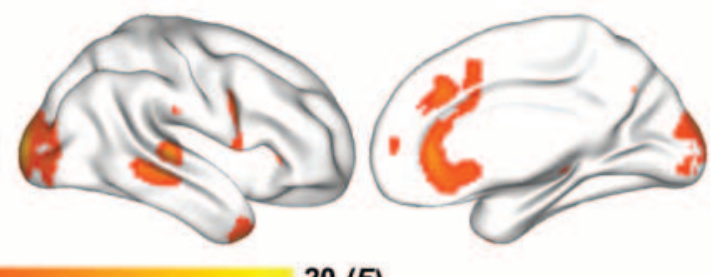

$20(F)$

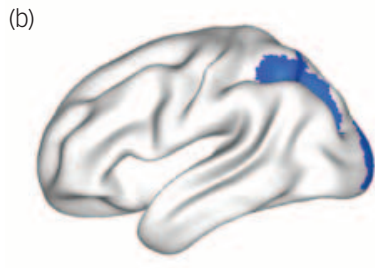

2.75

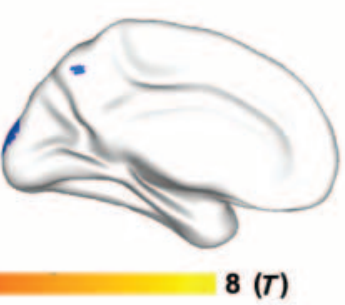

$8(T)$

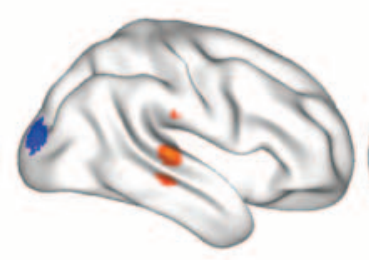

$-2.45$

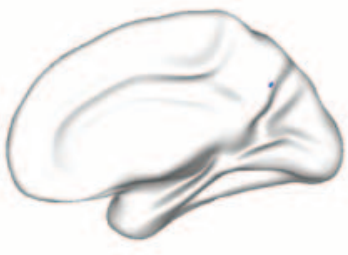

$-8(T)$

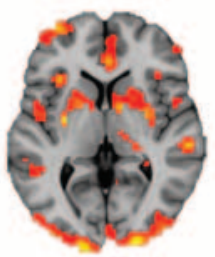

(c)

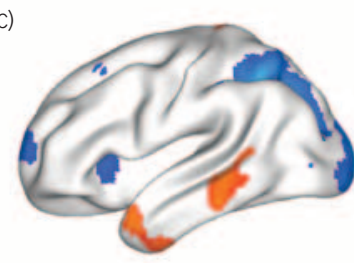

2.20

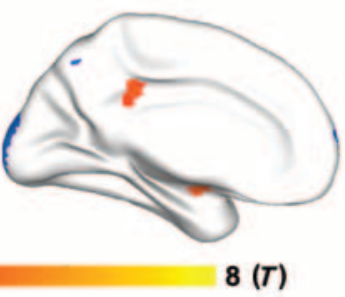

$8(T)$

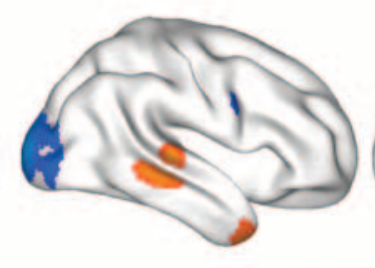

$-1.94$

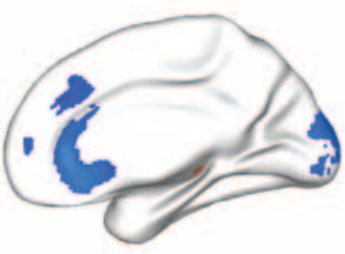

$-8(T)$
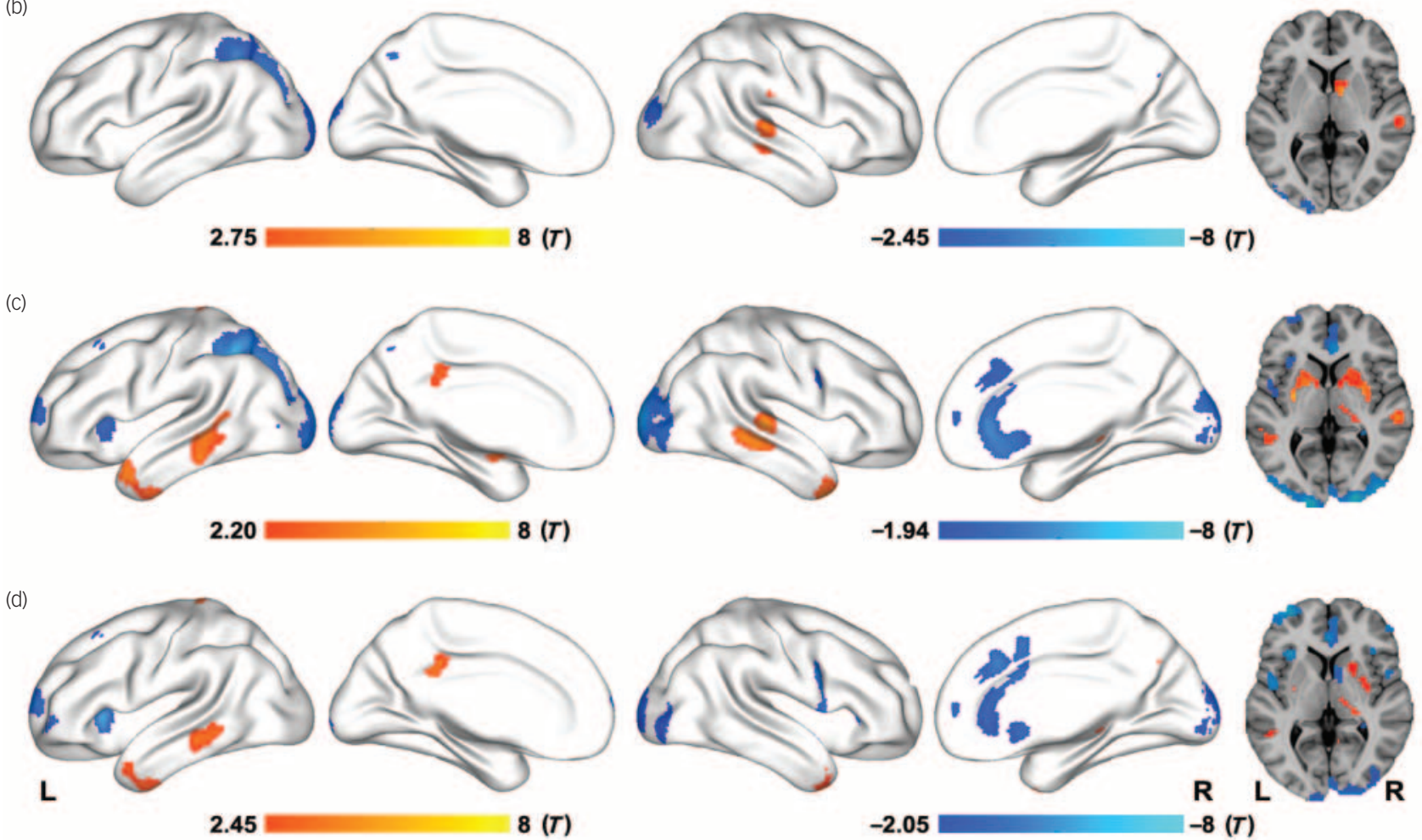

$-2.05$

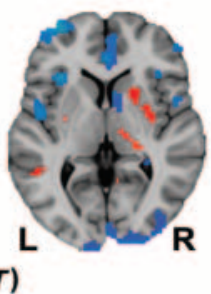

Fig 1. Brain regions exhibiting cerebral blood flow (CBF) differences among the auditory verbal hallucination (AVH), non-AVH and control groups.

(a) One-way analysis of covariance (ANCOVA) reveals brain regions with significant CBF differences among the three groups. Post hoc two-sample $t$-tests show brain regions with significant CBF differences between (b) the AVH and non-AVH groups, (c) the AVH and control groups, and (d) the non-AVH and control groups. The minimum values in the colour bars indicate statistical thresholds ( $F$ or $T$ ) of $P<0.05$ (false discovery rate (FDR) corrected). L, left; $R$, right.

volume in the right STG in schizophrenia, suggesting that volume reduction in the right STG is likely to be specific to the symptoms of AVHs. ${ }^{10}$ This paradox of functional and structural findings may indicate a disruption of the normal structure-function relationship or a decoupling between blood supply and brain structural topology. The left STG, including the primary and association auditory cortices, has been repeatedly found to play a prominent role in the aetiology of AVHs. ${ }^{7,8}$ The right STG is also implicated in auditory and language processing, particularly of the emotional and prosodic aspect of speech stimuli. ${ }^{46}$ In most right-handed people, the left hemisphere dominates over the right one in language production and perception. ${ }^{47}$ However, dysfunction of the right hemisphere language areas is capable of producing some single words or truncated sentences typically with a negative emotional content, ${ }^{48,49}$ for example swear words or terms of abuse. AVHs experienced by individuals with schizophrenia usually consist of these kinds of short phrases of low linguistic complexity, ${ }^{50,51}$ indicating that they may indeed be the product of the right language-related regions including the right STG. ${ }^{43}$ Another possible interpretation of our right lateralised findings is that according to the 'callosal relay' model, auditory signals must be dominantly transferred from the right hemisphere to the left side for processing. ${ }^{12,52,53}$ So it is conceivable that hyperfunction of the right STG may be capable of producing or amplifying AVH-related speech in the case of normal function of the left side. Converging evidence points towards the potential role of the caudate nucleus in the pathophysiology of AVHs. For example, several reports have shown that individuals with schizophrenia experiencing AVHs have significantly higher metabolic rates ${ }^{54}$ and grey matter volume ${ }^{55}$ in the caudate nucleus. Considering that the caudate nucleus has a central role in linguistic processes ${ }^{56}$ and regulating language switch, ${ }^{57,58}$ its dysfunction could be a key factor in the development and maintenance of AVHs. ${ }^{54}$

We also found decreased CBF in the parietal cortex in individuals with AVHs compared with individuals without AVHs and healthy controls. This cluster is located at the superior parietal lobule and the intra-parietal sulcus ${ }^{59-61}$ and is involved in top-down attention, working memory, motor coordination, numerical calculation, tool use and language. ${ }^{59,62.63}$ Most of these functions have been reported to be impaired in individuals with schizophrenia. ${ }^{64-67}$ Among these functions, the deficits in top-down attention ${ }^{68,69}$ and working memory ${ }^{70,71}$ are closely associated with AVHs. Thus, the reduced CBF in the superior 
(a)
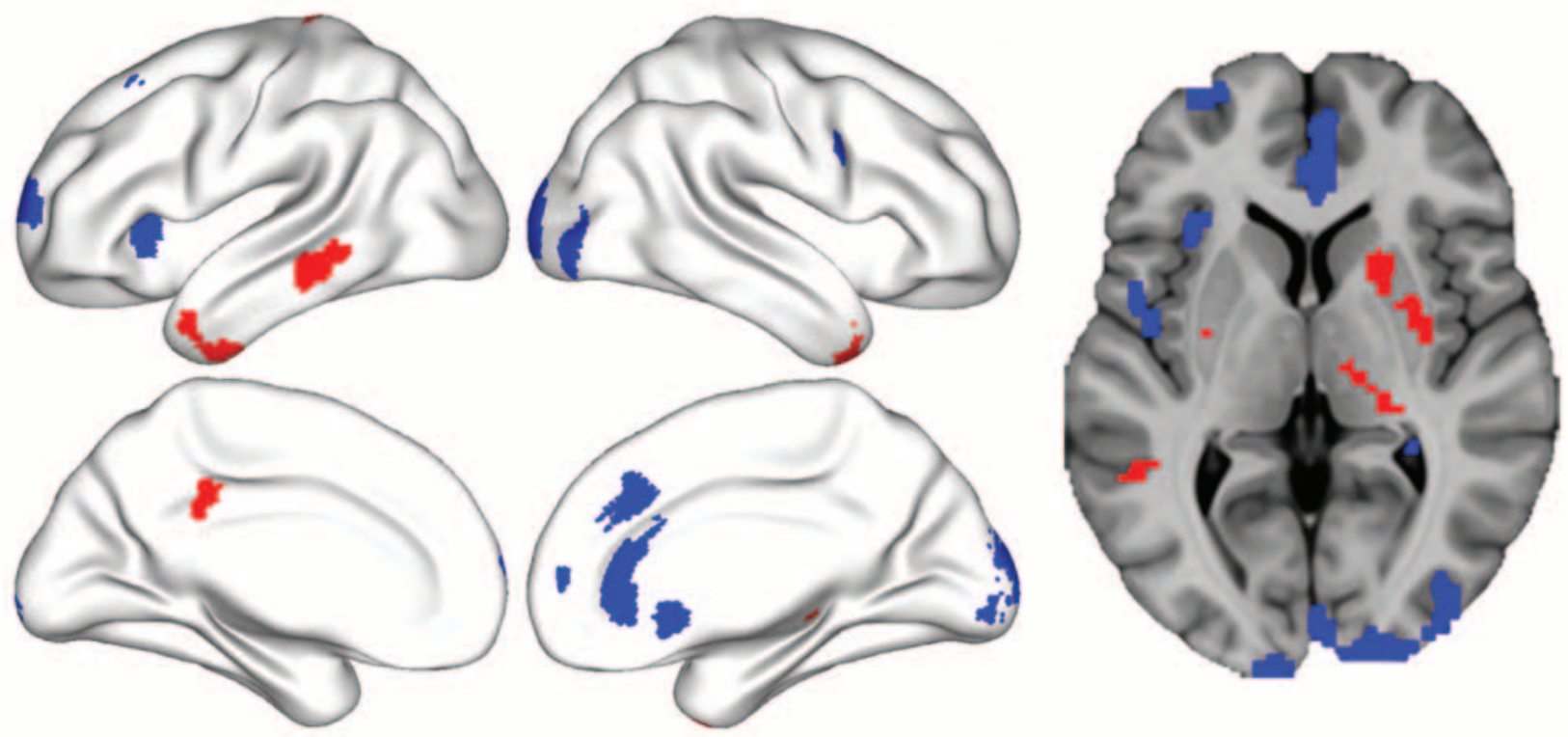

(b)
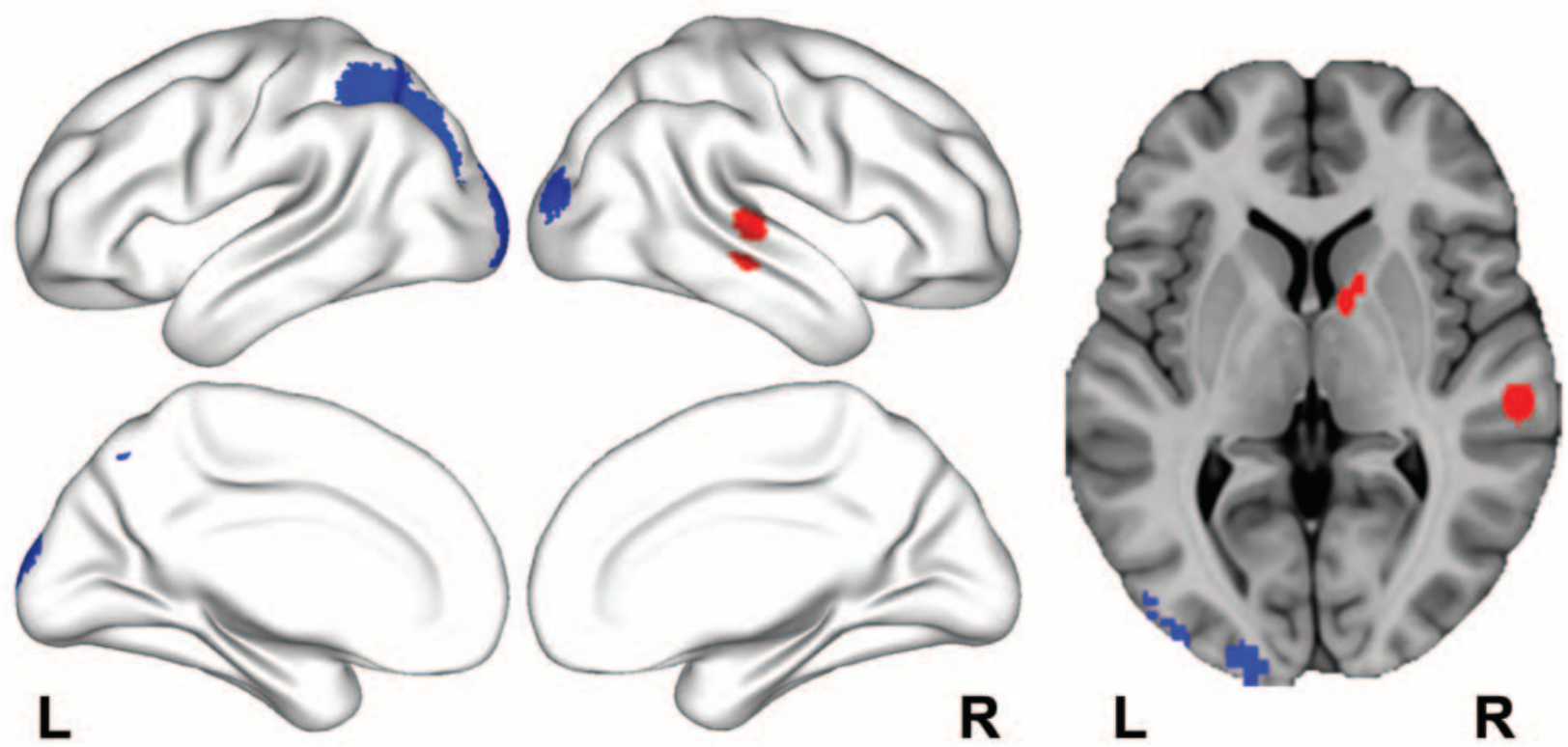

Fig. 2. Brain regions with (a) common and (b) exclusive cerebral blood flow (CBF) alterations in individuals with auditory verbal hallucinations (AVHS).

(a) Common CBF alterations in schizophrenia are defined as the overlapping regions between CBF-difference maps ( $P<0.05$, false discovery rate (FDR) Corrected) of the AVH-Control and non-AVH-Control groups (red represents CBF increase and blue means CBF reduction in both schizophrenia groups). (b) Exclusive CBF alterations are defined as the overlapping

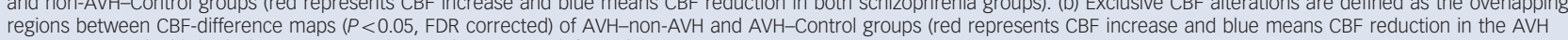
regions between CBF-difference maps ( $P<0.05$, FDR corrected) of AVH-non-AVH
group compared with both the non-AVH and control groups). L, left; R, right.

parietal lobule and the intra-parietal sulcus may be a candidate explanation for the attention and working memory deficits in individuals with schizophrenia with AVHs.

\section{Associations between CBF and AVH severity}

In our study, we did not find a correlation between CBF values of these AVH-exclusive brain areas and severity of auditory hallucinations assessed by the ARHS total score and frequency subscale in individuals with AVHs. These findings indicate that aberration of resting-state CBF may be a stable trait characteristic of AVH schizophrenia regardless of its severity. However, we cannot rule out the possibility that there is a complex relationship between $\mathrm{CBF}$ and AVH severity beyond a simple linear correlation. Thus, more complex models, such as a quadratic regression model, are needed to clarify the relationship between them.

\section{Limitations}

Several limitations must be noted when interpreting the results of the present study. First, the majority of the participates with schizophrenia received antipsychotic drug treatment and its effect on the CBF in schizophrenia is still unclear. Although the effect of this is likely to be relatively small, because there was no difference in antipsychotic dosage between the individuals with and without AVHs, future studies should focus on drug-naive individuals with schizophrenia to clarify the uncertainty. Second, we did not assess the psychotic symptoms, including AVHs, during the MRI scan, which means that some individuals with schizophrenia may have experienced AVHs and others may not while in the MRI scanner. Instead, we assessed AVHs and other symptoms of psychosis in all the individuals with schizophrenia before the MRI procedure. Third, individuals with schizophrenia who had not experienced 
AVHs within 12 months prior to MRI scanning were included in this study. These individuals may have experienced auditory hallucinations earlier in their psychotic illness, and they may still have trait characteristics of hallucinations. Finally, although the sample sizes of 35 individuals with AVHs and 41 individuals without AVHs are sufficient to compare intergroup differences, 35 individuals with AVHs might not be sufficient to investigate within-group correlation with symptom severity. This might be the reason for the lack of significant correlations between $\mathrm{CBF}$ and the AHRS scores. Future studies with a larger sample size of individuals with AVHs are needed to validate our findings.

\section{Implications}

In summary, in this study we present evidence that individuals with schizophrenia with and without AVHs share common CBF alterations in widespread brain regions including the frontal, temporal, occipital, insular and subcortical regions. More importantly, individuals with AVHs show exclusive $\mathrm{CBF}$ alterations consisting of an increase in the right auditory and striatal areas, and a reduction in the visual and parietal areas, indicating that there exists a CBF redistribution associated with AVHs.

\footnotetext{
Chuanjun Zhuo, MD, PhD, Department of Radiology and Tianjin Key Laboratory of Functional Imaging, Tianjin Medical University General Hospital, Tianjin, and Department of Psychiatry Functional Neuroimaging Laboratory, Tianjin Mental Health Center, Tianjin Anding Hospital, Tianjin; Jiajia Zhu, MD, Wen Qin, MD, Department of Radiology and Tianjin Key Laboratory of Functional Imaging, Tianjin Medical University General Hospital, China; Hongru Qu, MD, Xiaolei Ma, MD, Department of Psychiatry, Tianjin Anning Hospital, Tianjin, China; Chunshui Yu, MD, Department Psychiatry, Tianjin Anning Hospital, Tianjin, China; Chunshui Yu, MD, Department
of Radiology and Tianjin Key Laboratory of Functional Imaging, Tianjin Medical University General Hospital, Tianjin, China

Correspondence: Chunshui Yu, MD, Department of Radiology, Tianjin Medical University General Hospital, No. 154, Anshan Road, Heping District, Tianjin 300052, China. Email: chunshuiyu@tijmu.edu.cn

First received 31 Aug 2015, final revision 25 Sep 2016, accepted 7 Oct 2016
}

\section{Funding}

This study was supported by grants from the National Basic Research Program of China (973 program, 2011CB707801); Natural Science Foundation of China (91332113 and 81271551) and Tianjin Key Technology R\&D Program (14ZCZDSY00018).

\section{Reference}

1 Andreasen NC, Flaum M. Schizophrenia: the characteristic symptoms. Schizophr Bull 1991; 17: 27-49.

2 David AS. The cognitive neuropsychiatry of auditory verbal hallucinations: an overview. Cogn Neuropsychiatry 2004; 9: 107-23.

3 Hugdahl K. Auditory hallucinations: a review of the ERC "VOICE" project. World J Psychiatry 2015; 5: 193-209.

4 Gaite L, Vazquez-Barquero JL, Borra C, Ballesteros J, Schene A, Welcher B, et al. Quality of life in patients with schizophrenia in five European countries: the EPSILON study. Acta Psychiatr Scand 2002; 105: 283-92.

5 Shergill SS, Murray RM, McGuire PK. Auditory hallucinations: a review of psychological treatments. Schizophr Res 1998; 32: 137-50.

6 Jones SR. Do we need multiple models of auditory verbal hallucinations? Examining the phenomenological fit of cognitive and neurological models. Schizophr Bull 2010; 36: 566-75.

7 Allen P, Laroi F, McGuire PK, Aleman A. The hallucinating brain: a review of structural and functional neuroimaging studies of hallucinations. Neurosci Biobehav Rev 2008; 32: 175-91.

8 Allen P, Modinos G, Hubl D, Shields G, Cachia A, Jardri R, et al. Neuroimaging auditory hallucinations in schizophrenia: from neuroanatomy to neurochemistry and beyond. Schizophr Bull 2012; 38: 695-703.

9 Modinos G, Costafreda SG, van Tol MJ, McGuire PK, Aleman A, Allen P. Neuroanatomy of auditory verbal hallucinations in schizophrenia: a quantitative meta-analysis of voxel-based morphometry studies. Cortex 2013; 49: 1046-55.
10 Palaniyappan L, Balain V, Radua J, Liddle PF. Structural correlates of auditory hallucinations in schizophrenia: a meta-analysis. Schizophr Res 2012; 137: 169-73.

11 Geoffroy PA, Houenou J, Duhamel A, Amad A, De Weijer AD, Curcic-Blake B, et al. The arcuate fasciculus in auditory-verbal hallucinations: a meta-analysis of diffusion-tensor-imaging studies. Schizophr Res 2014; 159: 234-7.

12 Wigand M, Kubicki M, Clemm von Hohenberg C, Leicht G, Karch S, Eckbo R, et al. Auditory verbal hallucinations and the interhemispheric auditory pathway in chronic schizophrenia. World J Biol Psychiatry 2015; 16: 31-44.

13 Jardri R, Pouchet A, Pins D, Thomas P. Cortical activations during auditory verbal hallucinations in schizophrenia: a coordinate-based meta-analysis. Am J Psychiatry 2011; 168: 73-81.

14 Kuhn S, Gallinat J. Quantitative meta-analysis on state and trait aspects of auditory verbal hallucinations in schizophrenia. Schizophr Bull 2012; 38: 779-86.

15 Kompus K, Westerhausen R, Hugdahl K. The "paradoxical" engagement of the primary auditory cortex in patients with auditory verbal hallucinations: a meta-analysis of functional neuroimaging studies. Neuropsychologia 2011; 49: 3361-9.

16 Hoffman RE, Hampson M. Functional connectivity studies of patients with auditory verbal hallucinations. Front Hum Neurosci 2011; 6: 6.

17 Theberge J. Perfusion magnetic resonance imaging in psychiatry. Top Magn Reson Imag 2008; 19: 111-30.

18 Kindler J, Jann K, Homan $\mathrm{P}$, Hauf $\mathrm{M}$, Walther S, Strik W, et al. Static and dynamic characteristics of cerebral blood flow during the resting state in schizophrenia. Schizophr Bull 2015; 41: 163-70.

19 Liu J, Qiu M, Constable RT, Wexler BE. Does baseline cerebral blood flow affect task-related blood oxygenation level dependent response in schizophrenia? Schizophr Res 2012; 140: 143-8.

20 Pinkham A, Loughead J, Ruparel K, Wu WC, Overton E, Gur R, et al. Resting quantitative cerebral blood flow in schizophrenia measured by pulsed arterial spin labeling perfusion MRI. Psychiatry Res 2011; 194: 64-72.

21 Scheef L, Manka C, Daamen M, Kuhn KU, Maier W, Schild HH, et al. Resting-state perfusion in nonmedicated schizophrenic patients: a continuous arterial spin-labeling 3.0-T MR study. Radiology 2010; 256: 253-60.

22 Walther S, Federspiel A, Horn H, Razavi N, Wiest R, Dierks T, et al. Resting state cerebral blood flow and objective motor activity reveal basal ganglia dysfunction in schizophrenia. Psychiatry Res 2011; 192: 117-24.

23 Zhu J, Zhuo C, Qin W, Xu Y, Xu L, Liu X, et al. Altered resting-state cerebral blood flow and its connectivity in schizophrenia. J Psychiatr Res 2015; 63: 28-35.

24 Wolf ND, Gron G, Sambataro F, Vasic N, Frasch K, Schmid M, et al. Magnetic resonance perfusion imaging of auditory verbal hallucinations in patients with schizophrenia. Schizophr Res 2012; 134: 285-7.

25 First MB, Spitzer RL, Gibbon M, Williams JBW. Structured Clinical Interview for DSM-IV Axis I Disorders - Clinician Version (SCID-CV). American Psychiatric Press, 1997.

26 Kay SR, Fiszbein A, Opler LA. The positive and negative syndrome scale (PANSS) for schizophrenia. Schizophr Bull 1987; 13: 261-76.

27 Hoffman RE, Hawkins KA, Gueorguieva R, Boutros NN, Rachid F, Carroll K, et al. Transcranial magnetic stimulation of left temporoparietal cortex and medication-resistant auditory hallucinations. Arch Gen Psychiatry 2003; 60 : 49-56.

28 Andreasen NC. Methods for assessing positive and negative symptoms. Mod Probl Pharmacopsychiatry 1990; 24: 73-88.

29 Gardner DM, Murphy AL, O'Donnell H, Centorrino F, Baldessarini RJ. International consensus study of antipsychotic dosing. Am J Psychiatry 2010; 167: 686-93.

30 Moran LV, Sampath H, Kochunov P, Hong LE. Brain circuits that link schizophrenia to high risk of cigarette smoking. Schizophr Bull 2013; 39 1373-81.

31 Buxton RB, Frank LR, Wong EC, Siewert B, Warach S, Edelman RR. A general kinetic model for quantitative perfusion imaging with arterial spin labeling. Magn Reson Med 1998; 40: 383-96.

32 Xu G, Rowley HA, Wu G, Alsop DC, Shankaranarayanan A, Dowling M, et al. Reliability and precision of pseudo-continuous arterial spin labeling perfusion MRI on 3.0 T and comparison with 150-water PET in elderly subjects at risk for Alzheimer's disease. NMR Biomed 2010; 23: 286-93.

33 Friston KJ, Ashburner J, Kiebel SJ, Nichols TE, Penny WD. Statistical Parametric Mapping: The Analysis of Functional Brain Images. Academic Press, 2007

34 Aslan S, Lu H. On the sensitivity of ASL MRI in detecting regional differences in cerebral blood flow. Magn Reson Imaging 2010; 28: 928-35. 
35 Vercammen A, Knegtering $H$, Bruggeman R, Westenbroek HM, Jenner JA, Slooff $\mathrm{CJ}$, et al. Effects of bilateral repetitive transcranial magnetic stimulation on treatment resistant auditory-verbal hallucinations in schizophrenia: a randomized controlled trial. Schizophr Res 2009; 114: 172-9.

36 Gur RC, Gur RE. Hypofrontality in schizophrenia: RIP. Lancet 1995; 345 1383-4.

37 Manoliu A, Riedl V, Zherdin A, Muhlau M, Schwerthoffer D, Scherr M, et al Aberrant dependence of default mode/central executive network interactions on anterior insular salience network activity in schizophrenia. Schizophr Bull 2014; 40: 428-37.

38 Butler PD, Schechter I, Zemon V, Schwartz SG, Greenstein VC, Gordon J, et al. Dysfunction of early-stage visual processing in schizophrenia. Am J Psychiatry 2001; 158: 1126-33.

39 Byne W, Hazlett EA, Buchsbaum MS, Kemether E. The thalamus and schizophrenia: current status of research. Acta Neuropathologica 2009; 117: 347-68.

40 Onitsuka T, Shenton ME, Salisbury DF, Dickey CC, Kasai K, Toner SK, et al. Middle and inferior temporal gyrus gray matter volume abnormalities in chronic schizophrenia: an MRI study. Am J Psychiatry 2004; 161: 1603-11.

41 Perez-Costas E, Melendez-Ferro M, Roberts RC. Basal ganglia pathology in schizophrenia: dopamine connections and anomalies. J Neurochemistry 2010; 113: 287-302.

42 Sommer IE, de Weijer AD, Daalman K, Neggers SF, Somers M, Kahn RS, et al. Can fMRI-guidance improve the efficacy of rTMS treatment for auditory verbal hallucinations? Schizophr Res 2007; 93: 406-8.

43 Sommer IE, Diederen KM, Blom JD, Willems A, Kushan L, Slotema K, et al. Auditory verbal hallucinations predominantly activate the right inferior frontal area. Brain 2008; 131: 3169-77.

44 Woodruff PW, Wright IC, Bullmore ET, Brammer M, Howard RJ, Williams SC, et al. Auditory hallucinations and the temporal cortical response to speech in schizophrenia: a functional magnetic resonance imaging study. Am J Psychiatry 1997; 154: 1676-82.

45 van Swam C, Federspiel A, Hubl D, Wiest R, Boesch C, vermathen P, et al Possible dysregulation of cortical plasticity in auditory verbal hallucinations a cortical thickness study in schizophrenia. J Psychiatr Res 2012; 46 1015-23.

46 Downar J, Crawley AP, Mikulis DJ, Davis KD. A multimodal cortical network for the detection of changes in the sensory environment. Nature Neurosci 2000; 3: 277-83.

47 Knecht S, Drager B, Deppe M, Bobe L, Lohmann H, Floel A, et al. Handedness and hemispheric language dominance in healthy humans. Brain 2000; 123: 2512-8.

48 Strauss E, Wada J. Lateral preferences and cerebral speech dominance. Cortex 1983; 19: 165-77.

49 Van Lancker D, Cummings JL. Expletives: neurolinguistic and neurobehavioral perspectives on swearing. Brain Res Rev 1999; 31: 83-104.

50 Copolov D, Trauer T, Mackinnon A. On the non-significance of internal versus external auditory hallucinations. Schizophr Res 2004; 69: 1-6.

51 Stephane M, Thuras P, Nasrallah H, Georgopoulos AP. The internal structure of the phenomenology of auditory verbal hallucinations. Schizophr Res 2003 61: 185-93.

52 Hugdahl K, Carlsson G, Uvebrant P, Lundervold AJ. Dichotic-listening performance and intracarotid injections of amobarbital in children and adolescents. Preoperative and postoperative comparisons. Arch Neurol 1997; 54: $1494-500$.
53 Zaidel DW. Memory for scenes in stroke patients. Hemisphere processing of semantic organization in pictures. Brain 1986; 109: 547-60.

54 Horga G, Parellada E, Lomena F, Fernandez-Egea E, Mane A, Font M, et al. Differential brain glucose metabolic patterns in antipsychotic-naive firstepisode schizophrenia with and without auditory verbal hallucinations. J Psychiatry Neurosci 2011; 36: 312-21.

55 O'Daly OG, Frangou S, Chitnis X, Shergill SS. Brain structural changes in schizophrenia patients with persistent hallucinations. Psychiatry Res 2007; 156: 15-21.

56 Gil Robles S, Gatignol P, Capelle L, Mitchell MC, Duffau H. The role of dominant striatum in language: a study using intraoperative electrical stimulations. J Neurol Neurosurg Psychiatry 2005; 76: 940-6.

57 Abutalebi J, Annoni JM, Zimine I, Pegna AJ, Seghier ML, Lee-Jahnke H, et al. Language control and lexical competition in bilinguals: an event-related fMRI study. Cereb Cortex 2008; 18: 1496-505.

58 Crinion J, Turner R, Grogan A, Hanakawa T, Noppeney U, Devlin JT, et al. Language control in the bilingual brain. Science 2006; 312: 1537-40.

59 Humphreys GF, Lambon Ralph MA. Fusion and fission of cognitive functions in the human parietal cortex. Cereb Cortex 2015; 25: 3547-60.

60 Mars RB, Jbabdi S, Sallet J, O'Reilly JX, Croxson PL, Olivier E, et al. Diffusionweighted imaging tractography-based parcellation of the human parietal cortex and comparison with human and macaque resting-state functional connectivity. J Neurosci 2011; 31: 4087-100.

61 Nelson SM, Cohen AL, Power JD, Wig GS, Miezin FM, Wheeler ME, et al. A parcellation scheme for human left lateral parietal cortex. Neuron 2010; 67: $156-70$

62 Shomstein S. Cognitive functions of the posterior parietal cortex: top-down and bottom-up attentional control. Front Integr Neurosci 2012; 6: 38.

63 Koenigs M, Barbey AK, Postle BR, Grafman J. Superior parietal cortex is critical for the manipulation of information in working memory. $J$ Neurosci 2009; 29: 14980-6.

64 Walther S, Strik W. Motor symptoms and schizophrenia. Neuropsychobiology 2012; 66: 77-92.

65 Lee J, Park S. Working memory impairments in schizophrenia: a metaanalysis. J Abnorm Psychol 2005; 114: 599-611.

66 Neuhaus AH, Karl C, Hahn E, Trempler NR, Opgen-Rhein C, Urbanek C, et al. Dissection of early bottom-up and top-down deficits during visual attention in schizophrenia. Clin Neurophysiol 2011; 122: 90-8.

67 Covington MA, He C, Brown C, Naci L, McClain JT, Fjordbak BS, et al. Schizophrenia and the structure of language: the linguist's view. Schizophr Res 2005; 77: 85-98.

68 Loberg EM, Jorgensen HA, Kroken RA, Johnsen E. Auditory verbal hallucinations reflect stable auditory attention deficits: a prospective study. Cogn Neuropsychiatry 2015; 20: 81-94.

69 Papageorgiou C, Oulis P, Vasios C, Kontopantelis E, Uzunoglu N, Rabavilas A, et al. P300 alterations in schizophrenic patients experiencing auditory hallucinations. Eur Neuropsychopharmacol 2004; 14: 227-36.

70 Hoffman RE, Rapaport J, Mazure CM, Quinlan DM. Selective speech perception alterations in schizophrenic patients reporting hallucinated "voices". Am J Psychiatry 1999; 156: 393-9.

71 Gisselgard J, Anda LG, Bronnick K, Langeveld J, Ten Velden Hegelstad W, Joa I, et al. Verbal working memory deficits predict levels of auditory hallucination in first-episode psychosis. Schizophr Res 2014; 153: 38-41. 